\title{
BMJ Open Study protocol: randomised controlled trial evaluating exercise therapy as a supplemental treatment strategy in early multiple sclerosis: the Early Multiple Sclerosis Exercise Study (EMSES)
}

To cite: Riemenschneider M, Hvid LG, Ringgaard S, et al. Study protocol: randomised controlled trial evaluating exercise therapy as a supplemental treatment strategy in early multiple sclerosis: the Early Multiple Sclerosis Exercise Study (EMSES). BMJ Open 2021;11:e043699. doi:10.1136/ bmjopen-2020-043699

- Prepublication history and additional material for this paper is available online. To view these files, please visit the journal online (http://dx.doi.org/10. 1136/bmjopen-2020-043699).

Received 11 August 2020 Revised 15 December 2020 Accepted 31 December 2020

Check for updates

(C) Author(s) (or their employer(s)) 2021. Re-use permitted under CC BY-NC. No commercial re-use. See rights and permissions. Published by BMJ.

For numbered affiliations see end of article.

Correspondence to Mr Morten Riemenschneider; mori@ph.au.dk

\section{ABSTRACT}

Introduction In the relapsing remitting type of multiple sclerosis (MS) reducing relapses and neurodegeneration is crucial in halting the long-term impact of the disease. Medical disease-modifying treatments have proven effective, especially when introduced early in the disease course. However, patients still experience disease activity and disability progression, and therefore, supplemental early treatment strategies are warranted. Exercise appear to be one of the most promising supplemental treatment strategies, but a somewhat overlooked 'window of opportunity' exist early in the disease course. The objective of this study is to investigate exercise as a supplementary treatment strategy early in the disease course of MS. Methods and analysis The presented Early Multiple Sclerosis Exercise Study is a 48-week (plus 1-year follow-up) national multicentre single-blinded parallel group randomised controlled trial comparing two groups receiving usual care plus supervised high-intense exercise or plus health education (active control). Additionally, data will be compared with a population-based control group receiving usual care only obtained from the Danish MS Registry. The primary outcomes are annual relapse rate and MRI derived global brain atrophy. The secondary outcomes are disability progression, physical and cognitive function, MS-related symptoms, and exploratory MRI outcomes. All analyses will be performed as intention to treat.

Ethics and dissemination The study is approved by The Central Denmark Region Committees on Health Research Ethics (1-10-72-388-17) and registered at the Danish Data Protection Agency (2016-051-000001 (706)). All study findings will be published in scientific peer-reviewed journals and presented at relevant scientific conferences. Trial registration number NCT03322761.

\section{INTRODUCTION}

Multiple sclerosis (MS) is a chronic neurodegenerative disease of the central nervous system characterised by a complex pathogenesis and heterogeneous symptoms. Worldwide
Strengths and limitations of this study

- The Early Multiple Sclerosis Exercise Study is the first study specifically designed to investigate exercise therapy as a supplemental disease-modifying treatment strategy early in the disease course of multiple sclerosis

- A novel aspect of the present study is the addition of registry data from relevant patients forming a population-based control group, allowing assessment of the generalisability of study participants.

- With annual relapse rate being a primary outcome, the findings are limited to only apply for patients with the relapsing remitting type of multiple sclerosis.

- The present study holds the potential to change the current clinical practice regarding exercise therapy with multiple sclerosis by shifting the focus to early implementation of exercise as a supplemental treatment strategy.

approximately 2.2 million people are affected by MS, ${ }^{1}$ and most patients present with periodic neurological relapses. ${ }^{2}$ The number of relapses in the first years of the disease course have been shown to be associated with disability progression, with a higher number of relapses leading to a more rapid progression of disabilities. ${ }^{34}$ In addition to the acute neurological relapses, more diffuse neurodegeneration occurs from an early time point in the disease course, also leading to progression of disabilities. ${ }^{5}$ Therefore, a reduction in relapse rate and neurodegeneration early in the disease course is considered crucial in halting the long-term impact of the disease and is in most cases the primary aim of disease-modifying treatments (DMT). ${ }^{6}$

Within recent years, medical research has shown that initiation of DMT at an early stage 
is superior to initiation at a later stage in terms of reducing disease activity and disability progression. ${ }^{7-9}$ While these study findings clearly advocate for early medical treatment efforts, it is necessary to emphasise that patients still experience disease activity and develop neurological and functional deficits over time. ${ }^{56}$ Even from an early time point of the disease course, patients with MS show cognitive and functional deficits, ${ }^{10}$ suggesting an ongoing deterioration of the neurological (brain volume and cognitive function) and functional reserve capacity (physiological and functional capabilities). ${ }^{6} 11$ Noticeably, the medical DMTs have the potential to cause a wide range of adverse effects. ${ }^{12}$ 13 Consequently, supplementary (non-pharmacological) treatment strategies that can aid in reducing disease activity and disability progression are still warranted.

Exercise therapy appears to be one of the most potent supplementary (non-pharmacological) treatment strategies. Indeed, research evidence from the past 20 years has shown that exercise (1) is safe, tolerable and poses a limited risk of adverse events for patients with MS, ${ }^{14}$ (2) has numerous beneficial effects on general physical capacity and several MS-related symptoms, ${ }^{15}$ (3) likely holds a neuroprotective and disease-modifying potential $^{1617}$ and (4) may reduce the risk and/or postpone MS diagnosis. ${ }^{18}$ However, in a recent paper from our group, we pointed out that, despite the disease-modifying potential of exercise in MS, exercise is predominately viewed and used as symptomatic treatment. ${ }^{11}$ Consequently, exercise has generally been initiated rather late in the disease course ( $\geq 5$ years since diagnosis) based on existing research studies. ${ }^{11}$ This leaves an uninvestigated 'window of opportunity' for exercise therapy in the early phase treatment of MS, with the interesting potential to preserve the neurological and functional reserve at a higher level than medical treatment alone.

Therefore, the overall purpose of this study is to investigate exercise as a supplementary treatment strategy early in the disease course of MS. Specifically, we aim to investigate the effects of 48 weeks usual care plus a supervised progressive high intensity aerobic exercise intervention (48 weeks) compared with an control group receiving 48 weeks of usual care plus four health education sessions in relapsing remitting MS patients ( $\leq 2$ years since clinical diagnosis) primarily on disease activity (relapse rate) and measures of neurodegeneration (global brain atrophy), and secondarily on disability progression, physical and cognitive function and MS-related symptoms.

It is hypothesised that the patients receiving usual care plus supervised progressive high intensity exercise experience a lower relapse rate in the study period when compared with the control group receiving usual care plus health education and to population-based registry data from the Danish MS registry. Moreover, it is hypothesised that the global brain atrophy rate will be lower in the supervised exercise therapy group when compared with the health education control group. Secondarily, it is hypothesised that patients in the supervised exercise therapy group will have a lowered disability progression and a greater improvement in physical function, cognitive function and MS-related symptoms when compared with the health education control group.

\section{METHODS AND ANALYSIS \\ Study design}

The present study is a national multicentre single-blinded parallel group randomised controlled trial comparing a group receiving usual care plus supervised progressive high-intense exercise therapy to a control group receiving usual care plus health education. The study is currently ongoing, and the data collection is scheduled to be completed in March 2022. Assessors will be blinded to the analysis of the primary outcome (relapse rate) along with selected secondary outcomes (Expanded Disability Status Scale (EDSS) scoring, MRI outcomes and blood markers). The intervention period lasts 48 weeks with outcome assessment at baseline (T0), after 24 weeks (T1) and after 48 weeks (T2). The study also includes a follow-up period with outcome assessment 1 year after (T3) cessation of either the supervised exercise programme or the control condition with health education. In addition to the two randomised groups, data from a national populationbased control group receiving usual care only will be obtained from the Danish MS Registry. The total overview of the study design is depicted in figure 1 .

\section{Patient and public involvement}

Patients have not been involved in the design of this study, and will not be included in the recruitment or conduct of the study. Patients will only be involved as research participants. Individual feedback on their research participation will be given, and all participants will be invited to a lecture on the overall results of the project. Moreover, the results will be disseminated via the Danish MS Society.

\section{Participants}

Recruitment and eligibility

Patients with MS will be recruited via six Danish regional MS clinics (Aarhus University Hospital, Odense University Hospital, Clinics of Southern Denmark (Sønderborg, Esbjerg, Kolding), and Hospital Unit of Western Denmark), or via social media groups and events related to the Danish MS Society. In all cases, patients will be supplied with a leaflet explaining the rationale, design and content of the study and inviting them to participate. Those interested in participation will receive detailed written information as well as a leaflet from the National ethical committee explaining their rights as a participant in a health science research project. Furthermore, the project coordinator will contact participants by phone to explain the study, give the opportunity to ask questions, and to screen according to the inclusion criteria. Specifically, patients have to fulfil the following: (1) 18-60 years of age, (2) $\leq 2$ years since clinical diagnosis with relapsing remitting MS and (3) no relapses or changes 


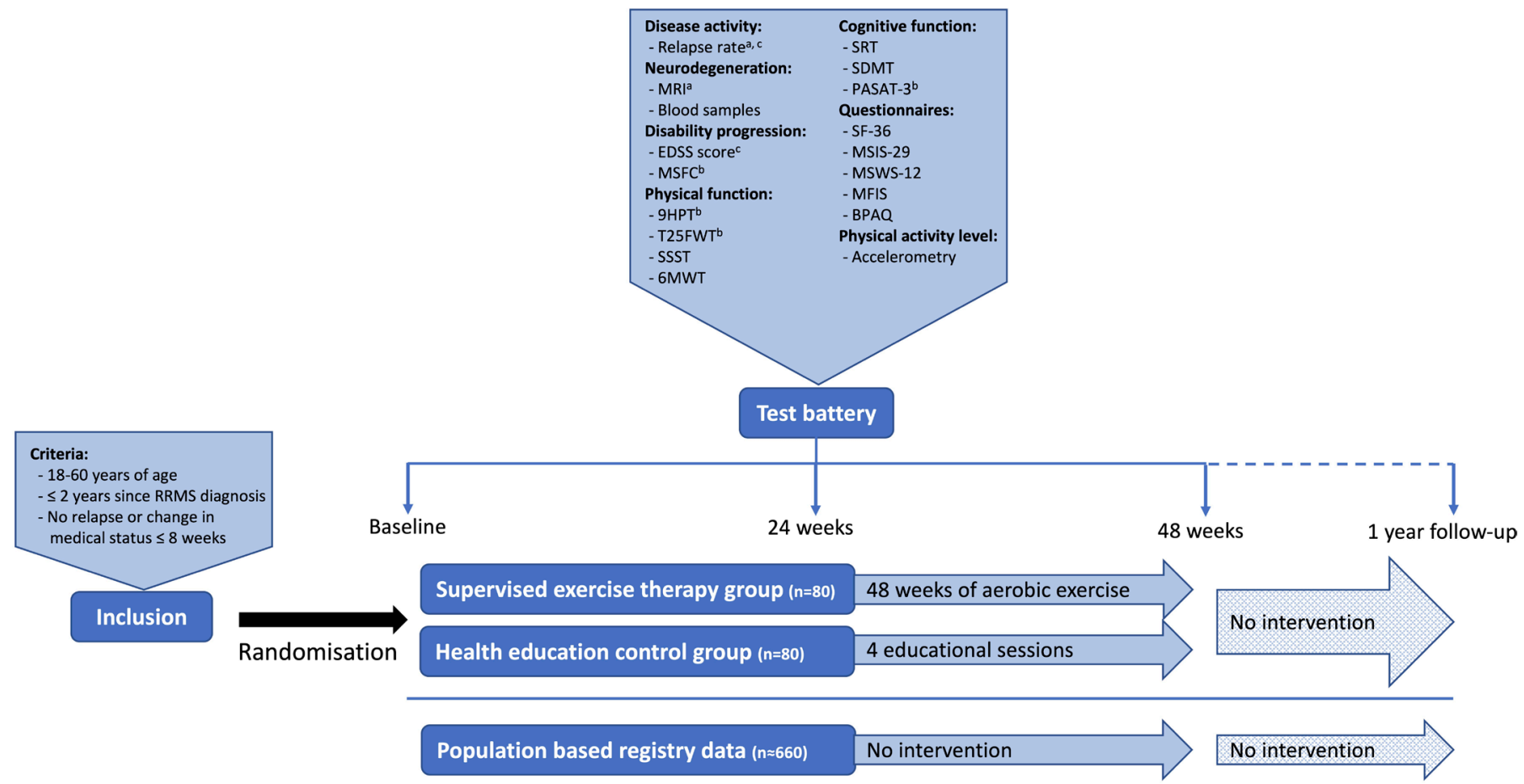

Figure 1 aprimary outcome, ${ }^{\mathrm{b}} \mathrm{Part}$ of the Multiple Sclerosis Functional Composite, ${ }^{\mathrm{c}}$ Outcomes available in the populationbased registry database. 6MWT, 6 min walk test; 9HPT, 9-Hole-Peg-Test; BPAQ, Baecke Physical Activity Questionnaire; EDSS, Expanded Disability Status Scale; MFIS, Modified Fatigue Impact Scale; MSFC, multiple sclerosis functional composite; MSIS29, Multiple Sclerosis Impact Scale 29 items; MSWS-12, Multiple Sclerosis Walking Scale 12 items; PASAT-3, paced auditory serial addition test $3 \mathrm{~s}$; RRMS, relapsing remitting multiple sclerosis; SDMT, symbol digit modality test; SF-36: Short Form 36; SRT, selective reminding test; SSST, six spot step test; T25FWT, timed 25-foot walk test.

in medication $\leq 8$ weeks prior to inclusion. Patients will be excluded if they: (1) are pregnant, or (2) have comorbidities or other issues thought to hinder participation in high intensity exercise activities. Finally, project nurses from the regional MS clinics confirm eligibility based on the patients' medical records, and patients sign informed consent (standard formula from the Danish National Research Ethics Committee, see online supplemental material 1 before inclusion.

\section{Sample size}

The calculation of the needed sample size is based on the estimated change in the primary outcomes, annualised relapse rate and global brain atrophy rate. A previous study has shown, that a health educational programme was unable to change any MS-related outcome measures over a period of 6 months in a group of patients with mild MS. ${ }^{19}$ On this basis, we expect no disease-modifying effects in the health education group (expected effect $=0$ ), enabling it to serve as a control group. Based on a report from Tallner et al showing a difference in annualised relapse rate of 0.325 between the patients with the highest and lowest levels of aerobic fitness (relapses over 2 years: high fitness level: $0.95 \pm 0.97$; low fitness level: $1.6 \pm 1.64)$, we expect disease-modifying effects in the supervised exercise therapy group corresponding to the group of patients with the highest levels of aerobic fitness versus the patients with the lowest fitness level. ${ }^{20}$ By using a statistical power $=0.80$ and a level of significance $=0.05$ and between-group change in annual relapse rate of $0.325 \pm 0.485$, calculations estimate that 80 patients should be included in each group (considering a $10 \%$ drop-out rate). In a subset of the patients, MRI scans will be conducted and for these outcomes, the global annual brain atrophy rate is the primary outcome. In a previous study from our group, an exercise-induced reduction of $0.27 \%$ in the global brain atrophy rate has been observed following 24 weeks of progressive resistance training (exercise: $-0.01 \% \pm 0.26$; control: $-0.28 \pm 0.52$ ) and based on that a sample size of 42 patients in each group is estimated for this outcome (again, considering a $10 \%$ drop-out rate). ${ }^{21}$

\section{Interventions}

\section{Random allocation procedure}

Randomisation to either the supervised exercise therapy group or the health education control group will be performed by the project manager (MR) in a 1:1 ratio and will be stratified by gender and line of treatment. The latter is in accordance with the Danish Medicine Council recommendations version 1.22019 (1) line treatment: dimethylfumerat, teriflunomide, glatirameracetat, interferon beta-1a, peg-interferon beta-1a, interferon beta-1b and (2) line treatment: natalizumab, ocrelizumab, fingolimod, cladribine). Allocation is concealed by the sealed envelope principle. 


\section{Supervised exercise therapy group}

Those randomised to the supervised exercise therapy group receive the exercise programme in addition to standard clinical treatment. The exercise intervention is designed and planned by the study exercise physiologists (MR, LGH and UD) and includes 2 weekly training sessions for a period of 48 weeks. Beside the supervised exercise the participants are left to self-administered behaviour. The test session after 24 weeks of training substitutes one of the 2 weekly training sessions, leaving a total of 95 training sessions to be completed. As patients are recruited from six MS clinics at hospitals throughout cities in the western part of Denmark, six training sites will be established in or near these same cities (ie, near each of the six recruiting MS clinics), in an attempt to facilitate adherence to the exercise intervention. Four of these training sites will be in facilities of four different physiotherapy schools, while two are in facilities of Sports Sciences at two Universities. After careful instruction from the study exercise physiologists, the training sessions are delivered and supervised by exercise physiology students, physiotherapy students or medical students. In addition to their educational background, all training supervisors are trained in delivering the specific exercise intervention and are provided oral and written safety instructions on exercise and MS (ie, Uhthoff's phenomenon and thermoregulation, and procedures in case of adverse events)..$^{22}$ Training sessions are offered in an 'open-house manner' 4 hours per day, 5 days a week. The training sessions are, therefore, performed either individually or in small groups, but never with a patient-supervisor ratio exceeding 4:1.

The foundation of the exercise therapy programme is generally based on four principles (adapted from Stathopoulos and Felson Duchan ${ }^{23}$ ): (1) individual tailoring, (2) application of specific exercises related to the goal of the programme, (3) progressive overload and (4) regular and continuous moderate-to-high intensity efforts to ensure the expected effects of the programme. On this basis, the exercise intervention is planned and monitored relative to the individual maximal heart rate (HR max) (Polar A300, Oulu, Finland) and composed of aerobic exercises, as this type of exercise have shown a substantial disease-modifying potential in the early phases of the disease course in the animal model of MS. ${ }^{16}$ In brief, the exercise therapy programme is composed of a mixture of continuous aerobic exercise sessions of 30-60 min duration at an intensity of $60 \%-80 \%$ of HR max and various interval training sessions of $30-60$ min total duration with an interval duration of 1-10 min and intensities varying from $65 \%-95 \%$ of HR max. In total, $3892 \mathrm{~min}$ of aerobic training are planned at an average intensity of $76.2 \%$ of HR max. The overall structure of the exercise intervention includes a periodisation into four meso cycles of 12 weeks with a planned progression within and between cycles to ensure a progressive overload and sustained high intensity efforts. The overall focus as well as the summarised total exercise duration and averaged intensity of each of the four cycles are presented in table 1 .

Generally, we do feel confident that the prescribed exercise programme is safe, feasible and effective in patients with MS as a recent study from our research group applying similar exercise sessions $(65 \%-95 \%$ HR max with similar work:rest ratios) have shown excellent adherence to exercise sessions (92.6 (6.9) \% completed sessions) and excellent compliance to the prescribed exercise intensity as well. ${ }^{24}$ In this study, some patients experienced difficulties in completing the regular intervals at $90 \%-95 \%$ HR max in the end of the intervention, and based on these experiences the exercise intensity is only progressed toward $90-95 \%$ HR max in a few short bouts in the study described in this protocol paper.

As the intensity of the exercise intervention is based solely relative to HR max, the mode of exercise (cycle ergometer, cross trainer, rowing ergometer, treadmill) is optional. Too see the detailed exercise intervention with the specific programme for each session, go to online supplemental material 2.

To report on the adherence to the exercise therapy programme the following data are registered by the training supervisor at each exercise session: (1) if the training is attended and completed, (2) exercise modalities, (3) duration of the session, (4) peak HR, (5) average HR for the total session, (6) HR at the last minute of

Table 1 Summary of the supervised exercise therapy intervention

\begin{tabular}{cclcc}
$\begin{array}{l}\text { Meso } \\
\text { cycle }\end{array}$ & $\begin{array}{l}\text { Sessions } \\
\text { (n) }\end{array}$ & Overall focus & $\begin{array}{l}\text { Total exercise } \\
\text { duration (minutes) }\end{array}$ & $\begin{array}{l}\text { Average intensity } \\
\text { (\% HR max) }\end{array}$ \\
\hline 1 & 24 & $\begin{array}{l}\text { Introduction and fundamental conditioning with a majority of } \\
\text { longer intervals and continuous exercise sessions. }\end{array}$ & 938 \\
2 & 24 & $\begin{array}{l}\text { Interval training with gradual, but alternating, increases in } \\
\text { duration and intensity and interspersed by continuous sessions. }\end{array}$ & 926 \\
3 & 23 & $\begin{array}{l}\text { Further improving of general conditioning and gradual } \\
\text { progression from longer intervals with moderate intensity to } \\
\text { shorter intervals with high intensity. } \\
\text { High intensity interval sessions interspersed by longer } \\
\text { continuous moderate intensity sessions. }\end{array}$ & 7027 \\
\hline
\end{tabular}

$\mathrm{HR}$, heart rate. 
each exercise bout to calculate the average exercising HR (without rest periods).

As a part of the exercise intervention, a watt-max test (similar to the one used for oxygen uptake $\left(\mathrm{VO}_{2}\right) \max$ testing, see below) is conducted on a cycle ergometer (Monark LC4, Vansbro, Sweden) at the end of the first and third meso cycle, respectively. This test is primarily conducted for motivational reasons, as it ensures that the participants have a short-term training goal and get regular feedback on the effect of the programme during each training meso cycle. At the same time, this enable a check of the HR max after each training cycle and subsequent adjustments of the prescribed intensity.

In case of clinical relapses, the clinical supervisor in the project (ES, neurologist and professor) will be consulted, and the further course will be determined together with the patient. Preferably, the patient will return to the exercise intervention, and if so, the time for the return to training will be determined by the clinical supervisor. When the patient return to the intervention the first 2 weeks of the exercise intervention (introduction and low to moderate intensity) will be repeated, and from that point the patient will progress from the stage of the exercise programme registered just before the clinical relapse. Of note, this will be at an intensity of no more than $70 \%$ of HR max (no matter the prescribed intensity). From this point, the intensity will on a weekly basis be increased by $5 \%$ of the HR max until the patient reach the intensity level prescribed in the original exercise intervention. In case of other adverse events, the same procedure will be followed.

\section{Health education control group}

Those randomised to the health educational programme follow standard clinical treatment and are offered four 1.5-hour sessions of health education throughout the 48 weeks study period. As a previous study showed no effect of a health educational programme on MS-related outcomes in a group of patients with mild MS, ${ }^{19}$ this is considered the control group of the randomised trial. The purpose of providing the four sessions is mainly to facilitate adherence to the control group, and as the health education programme is not matched in attention (time) to the intervention group and furthermore not expected to have an effect on MS-related outcomes this group is therefore not seen as an active comparison. All sessions are delivered by the same study employee (MR) and the four sessions are provided in small groups with one session every 12 weeks. The time frames and themes for each educational session are:

Session 1: delivered within weeks $0-4$ with the overall theme 'Physical activity'.

The participants are introduced to the definition of physical activity as well as the health benefits associated with complying to the guidelines on physical activity from the Danish Health Authorities (30 min of moderate-tovigorous physical activity each day, and $20 \mathrm{~min}$ of vigorous physical activity twice per week).
Session 2: delivered within weeks 12-16 with the overall theme 'aerobic training'.

The participants are introduced to the definition of aerobic training as well as the general and MS specific health benefits of aerobic training. ${ }^{165-27}$

Session 3: delivered within weeks 24-28 with the overall theme 'resistance training'.

The participants are introduced to the definition of resistance training as well as the general and MS specific health benefits of resistance training. ${ }^{21} 252829$

Session 4: delivered within weeks 36-40 with the overall theme 'Aalternative training'.

The participants are introduced to the definitions of yoga and Pilates as well as the general and MS specific health benefits of these types of activities. ${ }^{30} 31$

Besides the four educational sessions, the patients are left to self-administered behaviour.

\section{Population-based control data}

In addition to the two randomised groups of the study, data from the Danish MS registry will be obtained as population-based control data. The Danish MS registry started collecting data in $1948,{ }^{32} 33$ and a national database was established in 1996 including all patients with MS in Denmark receiving disease-modifying treatment, ${ }^{34}$ and this database was later affiliated to the Danish MS registry. The registry is a collaboration between the research programme 'Health and morbidity in Denmark' at the National Institute of Public Health and Copenhagen University Hospital, 'Rigshospitalet'. All cases are validated with reports from all neurological departments at Danish hospitals. Since 2015 the registry has been connected with the online database system, COMPOS, and the registry includes information on age, sex, time of diagnosis and first symptoms, disease course, treatment, selected clinical variables and date of death. ${ }^{35}$

In this study, data from all patients diagnosed with relapsing remitting MS within 2years from the 1 November 2018 will be extracted (approximately $\mathrm{n}=660$ patients available for data extraction). The data will be extracted covering the period 1 January 2019 to the 15 December 2019, and the data for the patients already included in one of the randomised intervention arms will be omitted. In this way, the registry-based cohort will match the inclusion criteria of the randomised trial and the period from which data will be extracted will have a similar duration. Therefore, the registry-based cohort will serve two purposes: (1) it allows us to evaluate whether the included patients in the randomised trial is a representative sample of the newly diagnosed ( $\leq 2$ years) patients in Denmark and (2) it allows us to compare the disease activity (annual relapse rate) and disability progression (EDSS progression) in the two groups in the randomised trial with this registry-based cohort (figure 1). Despite not being a part of the randomised design, these data can serve as important population-based usual care only control data as recruitment bias in exercise trials have previously been reported. ${ }^{36}$ 
Table 2 Overview of assessments and the order of testing at different time points

\begin{tabular}{lcccc} 
& Baseline & 24 weeks & 48 weeks & 1-year follow-up \\
\hline 1. MRI $^{*}$ & $\mathrm{X}$ & $\mathrm{X}$ & $\mathrm{X}$ & $\mathrm{X}$ \\
2. Resting blood sample & $\mathrm{X}$ & $\mathrm{X}$ & $\mathrm{X}$ & $\mathrm{X}$ \\
3. Cognition & & & & $\mathrm{X}$ \\
SRT & $\mathrm{X}$ & $\mathrm{X}$ & $\mathrm{X}$ & $\mathrm{X}$ \\
SDMT & $\mathrm{X}$ & $\mathrm{X}$ & $\mathrm{X}$ & $\mathrm{X}$ \\
PASAT-3 & $\mathrm{X}$ & $\mathrm{X}$ & $\mathrm{X}$ & $\mathrm{X}$ \\
SRT-delayed & $\mathrm{X}$ & $\mathrm{X}$ & $\mathrm{X}$ &
\end{tabular}

4. Physical function

\begin{tabular}{|c|c|c|c|c|}
\hline 9HРТ & $x$ & $x$ & $x$ & $x$ \\
\hline T25FWT & $x$ & $x$ & $x$ & $x$ \\
\hline SSST & $x$ & $x$ & $x$ & $x$ \\
\hline 6MWT & $x$ & $\mathrm{x}$ & $\mathrm{x}$ & $x$ \\
\hline \multicolumn{5}{|l|}{ 5. PROM's } \\
\hline Demographic form & $\mathrm{x}$ & & & \\
\hline SF-36 Health Survey & $x$ & $x$ & $\mathrm{x}$ & $x$ \\
\hline MSIS-29 & $x$ & $x$ & $\mathrm{x}$ & $x$ \\
\hline MSWS-12 & $x$ & $x$ & $x$ & $x$ \\
\hline MFIS & $x$ & $x$ & $x$ & $x$ \\
\hline Baecke Physical Activity & $x$ & & $x$ & $x$ \\
\hline 4 week exercise recall & $x$ & $x$ & $\mathrm{x}$ & $\mathrm{x}$ \\
\hline 6. Body composition & $x$ & $x$ & $x$ & $x$ \\
\hline 7. Aerobic fitness level & $x$ & $x$ & $x$ & $x$ \\
\hline 8. Accelerometry & $x$ & $x$ & $x$ & $x$ \\
\hline \multicolumn{5}{|l|}{ Clinical outcomes } \\
\hline Disease duration $†$ & $\mathrm{x}$ & & & \\
\hline Time since diagnosis $\dagger$ & $x$ & & & \\
\hline Annual relapse rate ${ }^{*} \dagger$ & & & $x$ & $\mathrm{x}$ \\
\hline EDSS score† & $\mathrm{x}$ & $\mathrm{x}$ & $\mathrm{x}$ & $x$ \\
\hline Change in medical DMT $\dagger$ & & $\mathrm{x}$ & $\mathrm{x}$ & $\mathrm{x}$ \\
\hline
\end{tabular}

All outcomes are assessed in both the supervised exercise therapy group and the health education control group. Outcomes marked with a *, is also assessed in the population-based control group.

*Primary outcome (MRl=global brain atrophy rate).

†Outcome measure also extracted from the Danish MS registry, forming the population-based control data.

DMT, disease-modifying therapy; EDSS, Expanded Disability Status Scale; 9HPT, 9-Hole Peg Test; MFIS, Modified Fatigue Impact Scale;

MSIS-29, Multiple Sclerosis Impact Scale 29-item; MSWS-12, Multiple Sclerosis Walking Scale 12-item; 6MWT, 6 min walk test; PASAT-3, paced auditory serial addition test (3 seconds version); PROM's, patient-reported outcome measures; SDMT, symbol digit modality test; SF36, Short-Form 36-item; SRT, selective reminding test; SSST, six spot step test; T25FWT, timed 25-foot walk test.

\section{Outcomes}

A summary of the test battery including the order of testing can be found in table 2. The details regarding each outcome are described in the following sections.

\section{Primary outcomes}

The overall primary outcomes of the study are annual relapse rate and MRI-derived annual global brain atrophy rate.

\section{Relapse rate}

The number of relapses in the study period is obtained from the medical records of the included patients. This will be done in collaboration with the respective MS clinics, and all relapses will be confirmed by a neurologist. According to the Danish national neurological association, a relapse is defined as new symptoms or worsening of existing symptoms, causing neurological dysfunction for a minimum of 24 hours without any signs of infection or fever. This exacerbation must be preceded by a stable 4 -week period. The annual relapse rate is calculated for each patient by dividing 365 days with the number of days in the study and multiplying by the number of relapses in the study period. Due to the clinically meaningful 
definition of this outcome any potential changes in the relapse rate will be considered clinically meaningful.

\section{Global brain atrophy}

All MRI scans will be performed on the same 3-Tesla MRI scanner (MAGNETOM Skyra, Siemens Medical Systems, Erlangen, Germany) at baseline, after 24 weeks, after 48 weeks and finally at 1-year follow-up. To limit the influence of time-of-day on brain volume measures patients are scanned at the same time of day $( \pm 2$ hours $)$ at each of the three timepoints. ${ }^{37}$ No specific hydration guideline is provided prior to the MRI acquisition but patients are encouraged to continue normal lifestyle and thus prepare for the three test days in similar ways, limiting the possible confounding effect of differences in hydration status between test days. ${ }^{38}$ The MRI scans will apply the same protocol as done in a previous study from our group, ${ }^{24}$ and the details are described in the following section as well as the section 'secondary MRI outcomes'.

To quantify global brain atrophy, structural $\mathrm{T} 1$ weighted (T1w) MP2RAGE images ${ }^{39}$ will be acquired with $\mathrm{TR}=5000$ $\mathrm{ms}, \mathrm{TE}=2.98 \mathrm{~ms}, \mathrm{TI}_{1}=700 \mathrm{~ms}, \mathrm{TI}_{2}=2500 \mathrm{~ms}$ at a spatial resolution of $1 \times 1 \times 1 \mathrm{~mm}^{3}$ and a matrix size of $256 \times 256 \times 176$. The T1w images will be processed using the longitudinal framework by Aubert-Broche et al and thereby images will be denoised, ${ }^{40}{ }^{41}$ bias field corrected, ${ }^{42}$ intensity normalised, linearly registered to Montreal Neurological Institute template (ICBM152) ${ }^{43}$ and skull stripped. ${ }^{44} \mathrm{~A}$ template specific to each subject will be created using the individual serial T1w images. ${ }^{45}$ These specific templates will moreover be nonlinearly registered to ICBM152 and segmented into grey matter (GM), white matter and cerebrospinal fluid (CSF) using an artificial neural network classifier. ${ }^{46}$ Finally, these segments will then be merged with the ICBM152 atlas for whole brain parcellation. On this basis, percentages of brain parenchymal fraction (BPF), GM parenchymal fraction, and WM parenchymal fraction (WMPF) can be obtained by taking the ratio of $\mathrm{GM}+\mathrm{WM}, \mathrm{GM}$ or WM, respectively, to the total brain intracranial volume, defined as the volume of GM+WM+CSF.

In addition to the quantification of global brain volume as BPF, the global brain volume will also be quantified using the automated longitudinal brain change analysis tool, SIENA, ${ }^{47}$ part of FSL. ${ }^{48}$ SIENA will be run with brain masks calculated by the longitudinal framework for improved sensitivity, ${ }^{49}$ as described above.

\section{Secondary outcomes \\ Disability progression}

Neurological impairments in MS can be assessed by grading the impairment of eight different functional systems (pyramidal, cerebellar, brain stem, sensory, bowel and bladder, visual, cerebral, other) to rate the total EDSS score. ${ }^{50}$ The EDSS score is considered the gold standard when assessing disability and disease progression in MS and it is used routinely as a clinical endpoint in trials of DMTs ${ }^{51}$ In this study, the EDSS score will be determined for each participant by trained neurologists at the six collaborative MS clinics during routine clinical visits. Therefore, the EDSS score nearest to the date of inclusion, to the date of completion, and to the date of 1 year since completion will be used to assess the progression of disabilities. However, recent studies have pointed out limitations in the EDSS score such as insufficient interrater and intrarater reproducibility and a low responsiveness-especially at the lower end of the scale ${ }^{52}$ Therefore, the main measure of disability progression will be the Multiple Sclerosis Functional Composite (MSFC).

The MSFC is a clinically meaningful measure of disability composed of three parts including measures of upper and lower extremity function as well as cognitive function, ${ }^{53}$ and this will be assessed at baseline, after 24 weeks, after 48 weeks, and again at 1-year follow-up. Specifically, the MSFC is comprised of the Timed 25-Foot Walk Test (T25FWT), the 9-Hole Peg Test (9HPT) and the Paced Auditory Serial Addition Test (PASAT). ${ }^{54}$

The T25FWT, a validated quantitative measure of lower extremity function and ambulation ${ }^{55}$ is completed on a clearly marked 25-foot course and patients are instructed to walk 25 feet as quickly as possible, but safely. ${ }^{56}$ The test is administered twice, and the average time (in seconds) of the two trials is registered. A change in walking speed in the T25FWT greater than $20 \%$ will be considered a clinically meaningful change. ${ }^{57}$

The 9HPT, a quantitative measure of manual dexterity and upper extremity function, is conducted with a 9HPT testing device (container, nine pegs, and nine holes in a square pattern). The patients are instructed to insert pegs one by one into each of the nine holes and then remove them again one by one, as fast as possible. The test is administered twice with both the dominant and non-dominant hand, and the average time (in seconds) of each hand is registered ${ }^{56} \mathrm{~A}$ change in manual dexterity function of more than $20 \%$ is considered a clinically meaningful change. ${ }^{58}$

The PASAT, a quantitative measure of cognitive function (auditory information processing speed and flexibility, and calculation ability) is conducted using an audio file presenting single digits every third second (in this case) for three consecutive minutes. The patients are instructed to add each new digit to the digit immediately prior to it, and the number of correct responses is the test result. ${ }^{56}$ To minimise a potential learning effect, two alternate forms of the PASAT will be used randomly. A change greater than $0.5 \mathrm{SD}$ of the studied population is considered a clinically meaningful change for the PASAT. ${ }^{59}$

Finally, to create the total MSFC-score, Z-scores for each component are created and averaged. The respective Z-scores will be calculated in accordance with the official scoring manual. ${ }^{56}$ In short, the preferred method in longitudinal clinical trials is to use test results from the baseline assessment of all patients in the study cohort, and then subtract the mean of the baseline measurements (from all patients) from the individual test result and dividing by the SD of the baseline measurements (from all patients). ${ }^{56}$ 


\section{Physical function}

In addition to the physical functional measures of the MSFC (T25FWT and 9HPT), the Six Spot Step Test (SSST) and the $6 \mathrm{~min}$ Walk Test (6MWT) will also be performed at baseline, after 24 weeks, after 48 weeks, and again at 1-year follow-up.

The SSST was developed in 2006 by Nieuwenhuis et al as a new measurement for walking ability in MS. ${ }^{60}$ The test is conducted on a $5 \mathrm{~m}$ rectangular course in a crisscross manner at fastest (although safe) speed. When performing the test patients are required to walk as fast as possible, while shoving five wooden blocks out of circles marked on the floor. This has to be done with one-foot alternating between the medial and lateral side of the foot. The test is conducted four times (two times with each foot), and the average time (in seconds) is registered. The test can be used as an alternative to the otherwise reliable and valid $\mathrm{T} 25 \mathrm{FWT}^{55}$ as the T25FWT have shown a low responsiveness due to its short conduction time and monotonous and automatised movement pattern. ${ }^{60}$ In addition, the SSST require a more complex movement pattern, including changes in direction, balance and coordination and the test have shown excellent construct validity and discriminative properties, ${ }^{61}$ as well as acceptable test-retest agreement and reliability. ${ }^{62}$

The $6 \mathrm{MWT}$ is a reliable and responsive outcome for assessment of walking capacity in patients with MS. ${ }^{63} 64$ The test will be performed in a $30 \mathrm{~m}$ hallway according to the script of Goldman et al. ${ }^{63}$ A change of more than 21.6 $\mathrm{m}$ in walking distance on the $6 \mathrm{MWT}$ will be considered a clinically meaningful change. ${ }^{64}$ Furthermore, the 6MWT also captures motor fatigability in patients with MS. ${ }^{65}$

In addition to the above functional measures, the aerobic fitness level of the patients will also be assessed at baseline, after 24 weeks, and again after 48 weeks. This will be done by an incremental exercise (watt-max) test on a bicycle ergometer (SRM, Jülich, Germany). The test will be conducted at a self-chosen cadence between 60 and 100 revolutions per minute with an initial workload of $45 \mathrm{~W}$ for females and $60 \mathrm{~W}$ for males. After a $5 \mathrm{~min}$ warm-up, the workload will be increased by $15 \mathrm{~W} / \mathrm{min}$ for females and $20 \mathrm{~W} / \mathrm{min}$ for males, until voluntary exhaustion. During the entire test expired gas will be collected in a mixing bag and the rate of $\mathrm{VO}_{2}$, rate of carbon dioxide release and respiratory exchange ratio will continuously be determined by an online respiratory gas exchange analyser (Oxigraf O2CPX, Oxigraf Inc., Sunnyvale California, USA). This will be expressed as $10 \mathrm{~s}$ averages and analysed by Innocor 8.10 software (Innovision, Glamsbjerg, Denmark). Prior to each exercise test, flow and gas analysers will be calibrated using a $3 \mathrm{~L}$ syringe (Hans Rudolph, series 5530) and a certified reference gas $\left(4.00 \% \mathrm{CO}_{2}\right.$ and $\left.16.50 \% \mathrm{O}_{2}\right)$ as described elsewhere. ${ }^{66}$ Also prior to each test, the patient's weight, fat mass and lean body mass will be measured (Tanita SC-330. Tokyo, Japan). HR will be monitored continuously during the test by a Polar HR monitor (Polar A300, Oulu, Finland), and patients will be verbally encouraged to continue as long as possible. From this, the HR max will be obtained and used to calculate the prescribed intensity of the exercise intervention. At the time of volitional exhaustion, the maximal workload, the time completed, and the perceived exhaustion (as of 6-20 Borg scale) will be noted. ${ }^{67}$ A similar incremental exercise test has previously been shown to be a valid and reliable measure of maximal $\mathrm{VO}_{2}$ in mild to moderately impaired patients with $\mathrm{MS},{ }^{66}$ and the maximal $\mathrm{VO}_{2}$ will be determined as the highest recorded average $\mathrm{VO}_{2}$ over a period of $30 \mathrm{~s}$. From that, the aerobic fitness level will be calculated and expressed as $\mathrm{mL} \mathrm{O}_{9} / \mathrm{kg}$ bodyweight/min.

\section{Cognitive function}

In addition to the PASAT, the Selective Reminding Test (SRT) and the Symbol Digit Modality Test (SDMT) will also be performed at baseline, after 24 weeks, and again after 48 weeks.

The SRT is a measure of verbal learning and memory, and is composed of the long-term storage score and the consistent long-term retrieval score. The test is a part of the Brief Repeatable Battery of Neuropsychological Tests (BRB-N), and will be conducted and scored according to the original instructions. ${ }^{68}$ After approximately $15 \mathrm{~min}$ (and after administration of the PASAT) patients will be asked to recall the word list, and the number of correct recalled words forms the delayed recall score. To minimise a potential learning effect, three alternate forms of the SRT will be used. A trained neuropsychologist makes these versions which are equivalent regarding the norms for word frequency.

The SDMT is a valid and reliable measure of cognitive processing speed and attention, and it is considered the gold standard when assessing processing speed in patients with MS. ${ }^{69}$ The test is also a part of the BRB-N and will be conducted and scored accordingly. ${ }^{68}$ Two versions of the SDMT will be used in a random order to minimise a potential learning effect. A change of four points or greater from baseline will be considered a clinically meaningful change in processing speed and attention. ${ }^{70}$

\section{Patient-reported outcome measures}

The patients will be asked to report their perception of different domains via several questionnaires.

As a multipurpose questionnaire assessing generic physical and mental health, the Short-Form Health Survey with 36 questions will be used. ${ }^{71}$ The questionnaire will be used in V.2.0 with a 4-week recall and according to the guidelines from the official user manual. ${ }^{72}$ Each item in the questionnaire is scored using norm-based T-scores and will be transformed to 0-100 range scores, according to the official guidelines. ${ }^{72}$ A Physical Component Summary and a Mental Component Summary will be obtained, ${ }^{73}$ and for both higher scores corresponds to better health status. To evaluate more disease-specific impact, the Multiple Sclerosis Impact Scale-29 (MSIS-29) will be applied.

The MSIS-29 measure physical and psychological impact of the disease from the patients' perspective and 
has been rated as the most robust (reliable and valid) patient-reported outcome measure in MS. ${ }^{74} 75$ The test is scored in a range of $1-5$ for each of the 29 questions, and a change in the MSIS-29 physical score of 7 points is considered a clinically meaningful change for patients with MS and an EDSS score between 0 and $5 .{ }^{76}$ Also assessing the impact of MS, but specifically on walking abilities and mobility, the Multiple Sclerosis Walking Scale-12 (MSWS12) will be used.77

The MSWS-12 is scored in a range of $1-5$ for each question and the total score will be transformed to a score ranging from 0 to 100 with a higher score corresponding to greater walking impairments. A change in MSWS-12 score of 10.7 points or greater is considered clinically meaningful. ${ }^{64}$ Importantly, the MSWS-12 is a more sensitive measure of walking related impairments than objective walking outcomes early in the disease course of MS. ${ }^{78}$

Fatigue is one of the most frequent and disabling symptoms of MS, and the impact of fatigue will be evaluated by the Modified Fatigue Impact Scale (MFIS) ${ }^{79}$ The MFIS score ranges from 0 to 84 , with higher scores indicating greater fatigue impact and a change ranging from 3.86 to 8.11 being clinically relevant. ${ }^{80}$

All of these questionnaires will be completed at baseline, after 24 weeks, after 48 weeks, and again at 1-year follow-up.

\section{Physical activity level}

Patients with MS have been reported to be substantially less physical active than matched healthy people. ${ }^{81} 82$ To objectively assess the level of physical activity all patients will wear a thigh-worn accelerometer (Axivity AX3, Axivity, Newcastle, UK) for seven consecutive days at baseline, after 24 weeks, after 48 weeks, and again at 1-year follow-up. The device will be attached to the self-reported weakest leg (mid-anterior thigh) with self-adhesive tape (Fixomull Stretch, BSN Medical, Hamburg, Germany). The raw data will be sampled at $100 \mathrm{~Hz}$ and with a sensitivity of $\pm 8 \mathrm{G}$. The setup of all accelerometers and the download of raw data will be conducted using OmGui V.1.0.0.30 (Newcastle University, UK). At baseline, no supervised exercise sessions or health education sessions will be completed within the 7 hours measuring period. The 7 hours measuring period after 24 weeks of intervention will be completed while the intervention is ongoing, and the assessment after 48 weeks of intervention will be completed in the week following the cessation of the intervention. In that way, potential changes in physical activity behaviour can be tracked and compared between study groups. Despite not being measured directly in patients with MS, objective measures of physical activity level by thigh-worn Axivity devices have proven to have a higher compliance and stronger validity than other placements, ${ }^{83}$ hence guiding the setup in this study. The data will be analysed using the vector magnitude counts in 60s epochs over an 18hours time span starting at 6:00 hour, resulting in an overall measure of physical activity: counts per minute (CPM). In addition, minutes of sedentary-light intensity, moderate intensity, and vigorous intensity physical activities will be determined according to relevant cut-off levels of CPM.

In addition to the objective accelerometry-based measure, physical activity will also be assessed by the Baecke Physical Activity Questionnaire at baseline, after 24 weeks, after 48 weeks and again at 1-year follow-up. ${ }^{84}$ This questionnaire quantifies the habitual physical activity level in three indexes: occupational (at work), sport (structured exercise) and leisure (leisure time). Each index is scored from 1 to 5 , with 5 indicating the highest level of physical activity. The questionnaire have a fair-to moderate validity, ${ }^{85}$ and interestingly patients with MS with higher scores in the Sport index have shown a lower relapse rate than patients with a lower score. ${ }^{20}$ The questionnaire is not validated in Danish; hence a nonvalidated Danish version will be used.

Lastly, all patients will also be asked to complete a retrospective exercise diary (4-week recall) at baseline, after 24 weeks, after 48 weeks and again at 1-year follow-up. Patients will have to report exercise modality, frequency, duration and intensity. The details of the retrospective exercise diary can be found in online supplemental material 3.

\section{Secondary MRI outcomes}

As stated previously, the MRI protocol is similar to the one used in a previous study from our research group, ${ }^{24}$ and the details for the secondary MRI outcomes will be explained in the following section.

T2-weighted fluid-attenuated inversion recovery (T2FLAIR) images will be acquired using a 3D sequence for assessing hyperintense T2 lesions with TR $=5000 \mathrm{~ms}$, $\mathrm{TE}=386 \mathrm{~ms}$, at a spatial resolution of $1.15 \times 1.15 \times 1.15$ $\mathrm{mm}^{3}$ and a matrix size of $192 \times 192 \times 144$. From the T1 and T2FLAIR images, T2 hyperintense lesions will be segmented by the lesion growth algorithm implemented in LST toolbox V.2.0.15 (www.statisticalmodelling.de/ lst.html) for SPM12 using the default settings. Moreover, T1 hypointense lesions (black holes) will be estimated by mapping the T1 relaxation times (T1-RT) for each voxel using the acquired images from MP2RAGE sequence and thresholding the T2 hyperintense lesion masks by those voxels with a T1-RT greater than or equal to $1500 \mathrm{~ms}{ }^{86}$ This threshold has been found to have the highest correlation with clinical disability. ${ }^{87}$

Diffusion kurtosis imaging ${ }^{88}$ will be acquired anteriorposterior and posterior-anterior using a 1-9-9 protocol with $\mathrm{TR}=10 \mathrm{~s}, \mathrm{TE}=97 \mathrm{~ms}$, at a spatial resolution of $2.67 \times 2.67 \times 2.70 \mathrm{~mm}^{3}$, a matrix size of $72 \times 72 \times 60$ and with $\mathrm{b}$ values of 0,1000 and $2500 \mathrm{~s} / \mathrm{mm}^{2}{ }^{89}{ }^{90}$ Images will be denoised, ${ }^{91}$ corrected for Gibbs ringing, ${ }^{92}$ eddy currents and subject movement. ${ }^{93}$ Diffusion kurtosis parameters including mean diffusivity, mean kurtosis tensor, fractional anisotropy and kurtosis fractional anisotropy, ${ }^{94}$ will be calculated using an inhouse pipeline (Center of Functionnaly Integrative Neuroscience, Aarhus University). Cortical thickness will be estimated using Fast 
Accurate Cortex Extraction (FACE) and the cortical surfaces produced by FACE will be used for surface based statistical analysis of the kurtosis parameters. ${ }^{95}{ }^{96}$ Specifically, $\mathrm{b}=0$ images will be linearly registered to the $\mathrm{T} 1 \mathrm{w}$ images and the outer and inner cortical surfaces from FACE will be transformed into diffusion kurtosis native space. ${ }^{97}$ To minimise the partial volume effect from WM and CSF a mid-cortical surface will be calculated. Interpolation and mapping to the mid-cortical surface of the diffusion kurtosis parameters will be performed for each subject, and in turn mapped to a template surface in MNI space to facilitate surface-based statistics. Parameters will be smoothed using a $20 \mathrm{~mm}$ full width half-maximum geodesic Gaussian kernel. The image masks of subcortical structures segmented on T1w images will be transformed to diffusion kurtosis imaging native space using the previously described transformation matrix and the mean kurtosis parameters will be calculated within each structure for all subjects. DKI analyses will be carried out in the following regions of interest (ROIs): cortex, hippocampus, thalamus, basal ganglia (defined as caudate, putamen and globus pallidus), and corpus callosum.

\section{Inflammatory and neurodegenerative biomarkers}

Blood samples will be collected in resting state at baseline, after 24 weeks, after 48 weeks, and again at 1-year follow-up. Patients will be allowed a minimum of $5 \mathrm{~min}$ supine rest before blood collection from the antecubital vein. Blood samples will be collected in ethylenediaminetetraacetic acid-treated tubes, resting for $90 \mathrm{~min}$, and subsequently centrifuged at $1200 \mathrm{~g}$ for $10 \mathrm{~min}$. Thereafter serum will be extracted and divided into five aliquots and stored at $-80^{\circ} \mathrm{C}$ until further analyses.

Analysis of blood samples will be exploratory and aim to investigate the effects of exercise on relevant biomarkers, such as Glial Fibrillar Acid Protein, ${ }^{98}$ proinflammatory and anti-inflammatory markers (eg, interleukin-6, interleukin-10, interleukin-17, tumour necrosis factor- $\alpha$ ), ${ }^{99}$ as well as neurodegenerative (eg, neurofilament light chain) and neurotrophic markers (eg, brain-derived neurotrophic factor, insulin-like growth factor). ${ }^{100} 101$

\section{Data handling and statistical analysis plan}

All data will undergo double data entry and be stored according to the guidelines from the Danish Data Protection Agency. No interim analysis is planned and such analysis will only be performed if the trial is stopped due to ethical reasons.

Based on baseline data, descriptive statistics will be used to present and compare characteristics of the study population across all three groups in the study. Moreover, the distributional properties of the data will be determined. The observations from baseline to 24 weeks and 48 weeks in the randomised controlled part of the study will be analysed using a multivariate repeated measurements mixed effects regression model with group and time as fixed effects. The population-based control data (from the Danish MS registry) on EDSS progression will be included in a separate multivariate repeated measurements mixed effects regression model controlling for a priori defined potential confounding variables: age, gender and time since diagnosis. Generally, the assumptions of multivariate normal distribution, equal variance and normal distribution of residuals will be checked. If data do not fulfil these basic assumptions, relevant transformation will be performed prior to analysis. All analyses of data from the randomised controlled part of the study will be performed as intention to treat and thereby include all patients tested after 48 weeks regardless of their compliance to the protocol. In case of low adherence to the supervised exercise intervention, a subanalysis will be performed per-protocol, excluding patients with adherence lower than $80 \%$ (calculated as the number of completed sessions divided by the number of planned sessions).

To compare the annual relapse rate between the supervised exercise therapy group, the health education control group and the population-based control group (data from the Danish MS registry) Poisson regression will be performed, controlling for a priori defined potential confounding variables: age, gender and time since diagnosis.

\section{Ethics and dissemination}

This study protocol (V.1.1, dated 18 October 2020) comply with the Standard Protocol Items: Recommendations for Interventional Trials statement, ${ }^{102}$ and study reporting will follow the guidelines of the Consolidated Standards of Reporting Trials statement. ${ }^{103}$ In addition, the CERT statement will be followed in relation to reporting the exercise intervention. ${ }^{104}$ The study is approved by The Central Denmark Region Committees on Health Research Ethics, ${ }^{1-10}$ registered at the Danish Data Protection Agency (2016-051-000001 (706)), and will conform to the principles of the Declaration of Helsinki. Any adverse events will be registered, assessed in collaboration with the clinical responsible (ES), and reported to the Central Denmark Region Committees on Health Research Ethics according to guidelines. The results of this study will be published by the investigators in relevant scientific peer-reviewed journals, no matter the study findings. Moreover, study results will be presented at relevant scientific conferences and communicated to patients through the Danish MS society.

\section{DISCUSSION AND PERSPECTIVES}

The presented study seeks to investigate supervised exercise as a supplemental treatment strategy early in the disease course of relapsing remitting MS. Effects of the intervention will primarily be investigated on measures of disease activity and neurodegeneration, and secondarily on measures of disability progression, cognitive and physical function, and symptoms of fatigue.

Efforts investigating the effects of exercise beyond rehabilitation (eg, as a supplemental disease-modifying 
strategy) are warranted, ${ }^{17} 105$ and an overlooked "window of opportunity' early in the disease course for MS exercise therapy have previously been identified. ${ }^{11}$ Importantly, the present study addresses both of these issues making the approach novel and innovative. Furthermore, the present study complies with the recommendations from recent publications guiding the field of MS rehabilitation and exercise aligning the methodology with the current stage of the literature. ${ }^{17} 106$ Specifically, the study has clearly defined primary outcomes and sample size calculations based hereupon (resulting in a large-scale exercise study), includes a rather long-term supervised exercise intervention and a well-monitored active control group (eg, importantly controlling the physical activity level of participants).

Another novel aspect of the present study is the addition of registry data from relevant patients forming a population-based control group. This allow us to assess the generalisability of the study and to compare this large-scale population-based group with the groups in the randomised part of the study. To our knowledge, this approach has not been applied previously in MS exercise research.

There are also some limitations to the presented study that should be kept in mind when interpreting the results. The sample size calculations include an expected dropout rate of $10 \%$, however, after termination of the inclusion for the study a systematic review and meta-analysis on adherence and drop-out in randomised controlled trials of exercise interventions in people with MS, reporting drop-out rates between $0 \%$ and $47 \% .{ }^{107}$ Therefore, there is a risk that the statistical power to detect true changes is reduced. One should also be aware, that the patients in this study are recruited based on time since diagnosis and in that sense the groups are homogeneous. However, as a diagnostic delay often is present the groups are not necessarily homogeneous in terms of disease duration defined by time since debut of symptoms. ${ }^{108}$ With relapse rate being a primary outcome of the present study, the findings naturally only apply for patients with relapsing remitting MS. Moreover, one should be cautious when interpreting the results on relapse rate as the annual relapse rate used to calculate the sample size in this study is higher than what is observed in the current DMT era. ${ }^{109}$ The study is, therefore, at risk of being powered based on an overestimated effect size and a subsequent low power to detect true changes. However, a recent study with annual relapse rates corresponding to the current DMT era report an exercise-induced reduction in annual relapse rate similar to what was used in the sample size calculation for this study (exercise: annual relapse rate $=0.00(0.00 ; 0.07)$ and control $0.45(0.28 ; 0.61)){ }^{24}$ The sample size calculated to undergo MRI in this study is based on the only study (at the time point when the study was designed and planned) providing data on the effects of exercise on MRI measures in MS, ${ }^{21}$ and in this study a somewhat counterintuitive brain atrophy rate of $0.01 \%$ was observed in the exercise group following 24 weeks of resistance training. Healthy individuals exhibit annual brain atrophy rates of $0.1 \%-0.3 \%$, and brain atrophy rates of $0.40 \%-0.52 \%$ is considered 'pathological' in patients with MS, when compared with healthy individuals. ${ }^{110}$ This somewhat counterintuitive brain atrophy rate observed in the exercise group in the study by Kjolhede et al may be explained by neuroplastic adaptations to exercise at molecular, cellular, functional and structural levels in patients undergoing a substantial behavioural change (ie, a longterm exercise regimen). ${ }^{101}$ However, this explanation is only speculative and the results regarding brain atrophy in the study presented in this protocol paper should therefore be interpreted cautiously as the study is at risk of being based on an overestimated effect size in the sample size calculation, and therefore, also at risk of having a low statistical power in detecting true changes. As all of the patients included in this study is diagnosed within 2 years and only have to be free of relapse and changes in DMT for 8 weeks prior to inclusion, there is a risk that changes in DMT prior to the study may affect MRI outcomes (eg, causing pseudoatrophy). ${ }^{111}$ The control group receiving four educational sessions (provided as a tool to facilitate adherence and with an expected null-effect on the primary outcomes) do not get the same level of attention. The population-based control group is limited to few clinical outcomes accessible from the database of the Danish MS registry, and can therefore not be compared across the full test battery. While this group mirrors usual care only, no information on their behaviour (ie, physical activity level and sports participation) is available, limiting the identification of 'active ingredients' in the changes on the selected outcomes in this group. Moreover, one has to be aware of the Hawthorne effect when interpreting the longitudinal observations from the registry data group, in comparison with the groups in the randomised controlled part of the study.

In summary, this is the first-ever study to investigate the effects of exercise in the very early stages of MS and thereby taking a more preventive approach aiming at lowering the disease activity more than medical DMTs alone, ${ }^{11}$ aiming at maintaining (or even improving) functional and neurological reserve capacity. ${ }^{17105}$ We expect the present study to hold the potential to change the current clinical practice regarding exercise therapy with MS. In particular, the present study may provide the first data supporting a warranted shift of paradigm where exercise will be considered a supplemental treatment strategy from an early timepoint in the disease course of MS. ${ }^{17}$ If these early exercise efforts show additional disease-modifying and neuroprotective effects, this is inherently of major interest to the individual MS patient, yet also to the healthcare system. While medical DMTs constitute the majority of healthcare costs for patients with mild MS, ${ }^{73}$ early exercise efforts may be a cost-effective supplemental treatment strategy to minimise disability progression and the huge-related costs. ${ }^{112} 113$ Another highly important perspective of early exercise efforts as a supplemental treatment strategy in MS is the improvement of general health of the patients and the derived reduction in the increased risk of lifestylerelated comorbidities observed in patients with MS. ${ }^{114}$ 


\section{Author affiliations}

${ }^{1}$ Exercise Biology, Department of Public Health, Aarhus University, Aarhus, Denmark

${ }^{2}$ The MR Research Centre, Aarhus University Hospital, Aarhus N, Denmark

${ }^{3}$ Center of Functionnally Integrative Neuroscience, Department of Clinical Medicine, Aarhus University, Aarhus, Denmark

${ }^{4}$ The Multiple Sclerosis Clinic, Department of Neurology, Aarhus University Hospital, Aarhus, Denmark

${ }^{5}$ Institute of Regional Health Research, University of Southern Denmark, Odense,

Denmark

${ }^{6}$ Department of Neurology, MS-Clinic of Southern Jutland (Sønderborg, Esbjerg, Kolding), Sønderborg, Denmark

Acknowledgements The authors thanks all participating patients as well as the collaborators in this national multicentre trial: MS clinics (Aarhus University Hospital, Odense University Hospital, Clinics of Southern Denmark (Sønderborg, Esbjerg, Kolding) and Hospital Unit of Western Denmark), Physiotherapy Schools (VIA University College (Holstebro, Haderslev, Esbjerg), University of Southern Denmark.

Contributors MR, LGH and UD drafted the manuscript. SR, MKEN and SE are discipline-specific supervisors and have contributed to the draft of specific sections in the manuscript. TP and ES are cosupervisors for the PhD training of MR. All authors have contributed to the revision of the draft and have read and approved the final version.

Funding The study is financed by Aarhus University, Faculty of Health (Vennelyst Boulevard 4, 8000 Aarhus C, DK, health@au.dk) and by the following external foundations: 'Trygfonden' (grant number: 123878), 'Augustinus fonden' (grant number: 17-2194), The Danish MS Society "Scleroseforeningen" (grant numbers: A33634 and A35468), 'Direktør Jacob Madsen og Hustru Olga Madsens Mindefond', 'Knud og Edith Eriksens Mindefond'.

Disclaimer None of the funders has a role in the design, management, analysis or interpretation of data, or writing and publication of results.

Competing interests MR declare no conflict of interests, LGH has received research support, travel grants, and/or teaching honoraria from Biogen and Sanofi Genzyme. SR declare no conflict of interests, MKEN declare no conflict of interests, SE declare no conflict of interests, TP declare no conflict of interests, ES declare no conflict of interests, UD has received research support, travel grants and/or teaching honoraria from Biogen Idec, Merck, Serono, Novartis, Bayer Schering, and Sanofi Aventis, as well as honoraria from serving on the scientific advisory boards of Biogen Idec and Genzyme.

Patient consent for publication Not required.

Provenance and peer review Not commissioned; externally peer reviewed.

Supplemental material This content has been supplied by the author(s). It has not been vetted by BMJ Publishing Group Limited (BMJ) and may not have been peer-reviewed. Any opinions or recommendations discussed are solely those of the author(s) and are not endorsed by BMJ. BMJ disclaims all liability and responsibility arising from any reliance placed on the content. Where the content includes any translated material, BMJ does not warrant the accuracy and reliability of the translations (including but not limited to local regulations, clinical guidelines, terminology, drug names and drug dosages), and is not responsible for any error and/or omissions arising from translation and adaptation or otherwise.

Open access This is an open access article distributed in accordance with the Creative Commons Attribution Non Commercial (CC BY-NC 4.0) license, which permits others to distribute, remix, adapt, build upon this work non-commercially, and license their derivative works on different terms, provided the original work is properly cited, appropriate credit is given, any changes made indicated, and the use is non-commercial. See: http://creativecommons.org/licenses/by-nc/4.0/.

ORCID iD

Morten Riemenschneider http://orcid.org/0000-0003-1903-0333

\section{REFERENCES}

1 GBD 2016 Multiple Sclerosis Collaborators. Global, regional, and national burden of multiple sclerosis 1990-2016: a systematic analysis for the global burden of disease study 2016. Lancet Neurol 2019;18:269-85.

2 Reich DS, Lucchinetti CF, Calabresi PA. Multiple sclerosis. $N$ Engl J Med 2018;378:169-80.
3 Lublin FD, Baier M, Cutter G. Effect of relapses on development of residual deficit in multiple sclerosis. Neurology 2003;61:1528-32.

4 Tremlett $\mathrm{H}$, Yousefi M, Devonshire V, et al. Impact of multiple sclerosis relapses on progression diminishes with time. Neurology 2009;73:1616-23.

5 Ziemssen T, De Stefano N, Sormani MP, et al. Optimizing therapy early in multiple sclerosis: an evidence-based view. Mult Scler Relat Disord 2015;4:460-9.

6 Giovannoni G, Butzkueven H, Dhib-Jalbut S, et al. Brain health: time matters in multiple sclerosis. Mult Scler Relat Disord 2016;9:S5-48.

7 Agius M, Meng X, Chin P, et al. Fingolimod therapy in early multiple sclerosis: an efficacy analysis of the transforms and freedoms studies by time since first symptom. CNS Neurosci Ther 2014:20:446-51.

8 Comi G, Filippi M, Barkhof F, et al. Effect of early interferon treatment on conversion to definite multiple sclerosis: a randomised study. The Lancet 2001;357:1576-82.

9 Chalmer TA, Baggesen LM, Nørgaard M, et al. Early versus later treatment start in multiple sclerosis: a register-based cohort study. Eur J Neurol 2018;25:1262-e110.

10 Thrue C, Riemenschneider M, Hvid LG, et al. Time matters: early-phase multiple sclerosis is accompanied by considerable impairments across multiple domains. Mult Scler 2020:135245852093623.

11 Riemenschneider M, Hvid LG, Stenager E, et al. Is there an overlooked "window of opportunity" in MS exercise therapy? Perspectives for early MS rehabilitation. Mult Scler 2018;24:886-94.

12 Rafiee Zadeh A, Askari M, Azadani NN, et al. Mechanism and adverse effects of multiple sclerosis drugs: a review article. Part 1. Int J Physiol Pathophysiol Pharmacol 2019;11:95-104.

13 Rafiee Zadeh A, Ghadimi K, Ataei A, et al. Mechanism and adverse effects of multiple sclerosis drugs: a review article. Part 2. Int $J$ Physiol Pathophysiol Pharmacol 2019;11:105-14.

14 Pilutti LA, Platta ME, Motl RW, et al. The safety of exercise training in multiple sclerosis: a systematic review. J Neurol Sci 2014;343:3-7.

15 Motl RW, Sandroff BM, Kwakkel G, et al. Exercise in patients with multiple sclerosis. Lancet Neurol 2017;16:848-56.

16 Souza PS, Gonçalves ED, Pedroso GS, et al. Physical exercise attenuates experimental autoimmune encephalomyelitis by inhibiting peripheral immune response and blood-brain barrier disruption. Mol Neurobiol 2017;54:4723-37.

17 Dalgas U, Langeskov-Christensen M, Stenager E, et al. Exercise as medicine in multiple Sclerosis-Time for a paradigm shift: preventive, symptomatic, and disease-modifying aspects and perspectives. Curr Neurol Neurosci Rep 2019;19:88

18 Dorans KS, Massa J, Chitnis T, et al. Physical activity and the incidence of multiple sclerosis. Neurology 2016;87:1770-6.

19 Feys P, Tytgat K, Gijbels D, et al. Effects of an 1-day education program on physical functioning, activity and quality of life in community living persons with multiple sclerosis. NeuroRehabilitation 2013;33:439-48.

20 Tallner A, Waschbisch A, Wenny I, et al. Multiple sclerosis relapses are not associated with exercise. Mult Scler 2012;18:232-5.

21 Kjolhede T, Siemonsen S, Wenzel D, et al. Can resistance training impact MRI outcomes in relapsing-remitting multiple sclerosis? Mult Scler 2017; 1352458517722645.

22 Opara JA, Brola W, Wylegala AA, et al. Uhthoff's phenomenon 125 years later - what do we know today? J Med Life 2016;9:101-5.

23 Stathopoulos E, Felson Duchan J. History and principles of exercise-based therapy: how they inform our current treatment. Semin Speech Lang 2006;27:227-35.

24 Langeskov-Christensen M, Grøndahl Hvid L, Nygaard MKE, et al. Efficacy of high-intensity aerobic exercise on brain MR measures in multiple sclerosis. Neurology 2020:10.1212/ WNL.0000000000011241.

25 McArdle W. Exercise physiology : energy, nutrition, and human performance. Philadelphia: Wolters Kluwer/Lippincott Williams \& Wilkins Health, 2010.

26 Langeskov-Christensen M, Heine M, Kwakkel G, et al. Aerobic capacity in persons with multiple sclerosis: a systematic review and meta-analysis. Sports Med 2015;45:905-23.

27 Heine M, Port vande I, Rietberg MB, et al. Exercise therapy for fatigue in multiple sclerosis. Cochrane Database Syst Rev 2015;9:CD009956.

28 Kjølhede T, Vissing K, de Place L, et al. Neuromuscular adaptations to long-term progressive resistance training translates to improved functional capacity for people with multiple sclerosis and is maintained at follow-up. Mult Scler 2015;21:599-611. 
29 Dalgas U, Stenager E, Jakobsen J, et al. Resistance training improves muscle strength and functional capacity in multiple sclerosis. Neurology 2009;73:1478-84.

30 Mooventhan A, Nivethitha L. Evidence based effects of yoga in neurological disorders. J Clin Neurosci 2017;43:61-7.

31 Sánchez-Lastra MA, Martínez-Aldao D, Molina AJ, et al. Pilates for people with multiple sclerosis: a systematic review and metaanalysis. Mult Scler Relat Disord 2019;28:199-212.

32 Koch-Henriksen N. The Danish multiple sclerosis registry: a 50-year follow-up. Mult Scler 1999;5:293-6.

33 Brønnum-Hansen H, Koch-Henriksen N, Stenager E. The Danish multiple sclerosis registry. Scand J Public Health 2011;39:62-4.

34 Koch-Henriksen N, Sørensen PS. The Danish national project of interferon-beta treatment in relapsing-remitting multiple sclerosis. The Danish multiple sclerosis group. Mult Scler 2000;6:172-5.

35 Registry TDMS. About the registry. Available: https://www. rigshospitalet.dk/english/departments/neuroscience-centre/ department-of-neurology/research/the-danish-multiple-sclerosisregistry/about-the-registry/Pages/about-the-danish-multiplesclerosis-registry.aspx

36 Cooke R, Jones A. Recruiting adult participants to physical activity intervention studies using sport: a systematic review. BMJ Open Sport Exerc Med 2017;3:e000231.

37 Nakamura K, Brown RA, Narayanan S. Alzheimer's Disease Neuroimaging I. Diurnal fluctuations in brain volume: Statistical analyses of MRI from large populations. Neuroimage 2015;118:126-32.

38 Nakamura K, Brown RA, Araujo D, et al. Correlation between brain volume change and $\mathrm{T} 2$ relaxation time induced by dehydration and rehydration: implications for monitoring atrophy in clinical studies. Neuroimage 2014;6:166-70.

39 Marques JP, Kober T, Krueger G, et al. MP2RAGE, a self bias-field corrected sequence for improved segmentation and T1-mapping at high field. Neuroimage 2010;49:1271-81.

40 Aubert-Broche B, Fonov VS, García-Lorenzo D, et al. A new method for structural volume analysis of longitudinal brain MRI data and its application in studying the growth trajectories of anatomical brain structures in childhood. Neuroimage 2013;82:393-402.

41 Coupe P, Yger P, Prima S, et al. An optimized blockwise nonlocal means denoising filter for $3-D$ magnetic resonance images. IEEE Trans Med Imaging 2008;27:425-41.

42 Sled JG, Zijdenbos AP, Evans AC. A nonparametric method for automatic correction of intensity nonuniformity in MRI data. IEEE Trans Med Imaging 1998;17:87-97.

43 Collins DL, Neelin P, Peters TM, et al. Automatic 3D intersubject registration of Mr volumetric data in standardized Talairach space. $J$ Comput Assist Tomogr 1994;18:192-205.

44 Eskildsen SF, Coupé P, Fonov V, et al. Beast: brain extraction based on nonlocal segmentation technique. Neuroimage 2012;59:2362-73.

45 Fonov V, Evans AC, Botteron K, et al. Unbiased average age-appropriate atlases for pediatric studies. Neuroimage 2011;54:313-27.

46 Zijdenbos A, Forghani R, Evans A, eds. Automatic quantification of $M S$ lesions in $3 D \mathrm{MRI}$ brain data sets: validation of INSECT. Berlin, Heidelberg: Springer Berlin Heidelberg, 1998.

47 Smith SM, Zhang Y, Jenkinson M, et al. Accurate, robust, and automated longitudinal and cross-sectional brain change analysis. Neuroimage 2002;17:479-89.

48 Smith SM, Jenkinson M, Woolrich MW, et al. Advances in functional and structural Mr image analysis and implementation as fsl. Neuroimage 2004;23 Suppl 1:S208-19.

49 Nakamura K, Eskildsen SF, Narayanan S, et al. Alzheimer's Disease Neuroimaging I. Improving the SIENA performance using BEaST brain extraction. PLoS One 2018;13:e0196945.

50 Kurtzke JF. Rating neurologic impairment in multiple sclerosis: an expanded disability status scale (EDSS). Neurology 1983;33:1444-52.

51 Kragt JJ, Nielsen JM, van der Linden FAH, et al. How similar are commonly combined criteria for EDSS progression in multiple sclerosis? Mult Scler 2006;12:782-6.

52 Hobart J, Freeman J, Thompson A. Kurtzke scales revisited: the application of psychometric methods to clinical intuition. Brain 2000;123:1027-40.

53 Polman $\mathrm{CH}$, Rudick RA. The multiple sclerosis functional composite: a clinically meaningful measure of disability. Neurology 2010;74:S8-15

54 Fischer JS, Rudick RA, Cutter GR, et al. The multiple sclerosis functional composite measure (MSFC): an integrated approach to MS clinical outcome assessment. National MS Society clinical outcomes assessment Task force. Mult Scler 1999;5:244-50.
55 Motl RW, Cohen JA, Benedict R, et al. Validity of the timed 25-foot walk as an ambulatory performance outcome measure for multiple sclerosis. Mult Scler 2017;23:704-10.

56 Fischer JS, Jak AJ, Kniker JE, et al. Multiple sclerosis functional composite (MSFC): administration and scoring manual. New York: National Multiple Sclerosis Society, 2001.

57 Hobart J, Blight AR, Goodman A, et al. Timed 25-foot walk: direct evidence that improving $20 \%$ or greater is clinically meaningful in MS. Neurology 2013;80:1509-17.

58 Feys P, Lamers I, Francis G, et al. The Nine-Hole PEG test as a manual dexterity performance measure for multiple sclerosis. Mult Scler 2017;23:711-20.

59 Hoffmann-La Roche L. Clinical Review Report: Ocrelizumab (Ocrevus):I ndication: Management of adult patients with early primary progressive multiple sclerosis as defined by disease duration and level of disability, in conjunction with imaging features characteristic of inflammatory activity. Canada: Canadian Agency for Drugs and Technologies in Health, 2018.

60 Nieuwenhuis MM, Van Tongeren H, Sørensen PS, et al. The six spot step test: a new measurement for walking ability in multiple sclerosis. Mult Scler 2006;12:495-500.

61 Sandroff BM, Motl RW, Sosnoff JJ, et al. Further validation of the Six-Spot step test as a measure of ambulation in multiple sclerosis. Gait Posture 2015;41:222-7.

62 Callesen J, Richter C, Kristensen C, et al. Test-retest agreement and reliability of the six spot step test in persons with multiple sclerosis. Mult Scler 2019;25:286-94.

63 Goldman MD, Marrie RA, Cohen JA. Evaluation of the six-minute walk in multiple sclerosis subjects and healthy controls. Mult Scler 2008;14:383-90.

64 Baert I, Freeman J, Smedal T, et al. Responsiveness and clinically meaningful improvement, according to disability level, of five walking measures after rehabilitation in multiple sclerosis. Neurorehabil Neural Repair 2014;28:621-31.

65 Leone C, Severijns D, Dolezalova V, et al. Prevalence of WalkingRelated motor fatigue in persons with multiple sclerosis: decline in walking distance induced by the 6-minute walk test. Neurorehabil Neural Repair 2016;30:373-83.

66 Langeskov-Christensen M, Langeskov-Christensen D, Overgaard K, et al. Validity and reliability of VO2-max measurements in persons with multiple sclerosis. J Neurol Sci 2014;342:79-87.

67 Borg GA. Psychophysical bases of perceived exertion. Med Sci Sports Exerc 1982;14:377-81.

68 Boringa JB, Lazeron RHC, Reuling IEW, et al. The brief repeatable battery of neuropsychological tests: normative values allow application in multiple sclerosis clinical practice. Mult Scler 2001;7:263-7.

69 Benedict RHB, DeLuca J, Phillips G, et al. Validity of the symbol digit modalities test as a cognition performance outcome measure for multiple sclerosis. Mult Scler 2017;23:721-33.

70 Strober L, DeLuca J, Benedict RHB, et al. Symbol digit modalities test: a valid clinical trial endpoint for measuring cognition in multiple sclerosis. Mult Scler 2019;25:1781-90.

71 Ware JE. Sf-36 health survey update. Spine 2000;25:3130-9.

72 Maruish ME, Kosinski M, Bjorner JB, et al. User's manual for the SF$36 v 2$ health survey (3rd ed. Lincoln, RI: QualityMetric Incorporated, 2011.

73 Kobelt G, Thompson A, Berg J, et al. New insights into the burden and costs of multiple sclerosis in Europe. Mult Scler 2017;23:1123-36.

74 Hobart J, Lamping D, Fitzpatrick R. The multiple sclerosis impact scale (MSIS-29): a new patient-based outcome measure. Brain 2001;124:962-73.

75 Khurana V, Sharma H, Afroz N, et al. Patient-Reported outcomes in multiple sclerosis: a systematic comparison of available measures. Eur J Neurol 2017;24:1099-107.

76 Costelloe L, O'Rourke K, Kearney H, et al. The patient knows best: significant change in the physical component of the multiple sclerosis impact scale (MSIS-29 physical). J Neurol Neurosurg Psychiatry 2007;78:841-4.

77 Hobart JC, Riazi A, Lamping DL, et al. Measuring the impact of MS on walking ability: the 12-Item MS walking scale (MSWS-12). Neurology 2003;60:31-6.

78 Langeskov-Christensen D, Feys P, Baert I, et al. Performed and perceived walking ability in relation to the expanded disability status scale in persons with multiple sclerosis. J Neurol Sci 2017;382:131-6.

79 Fisk JD, Ritvo PG, Ross L, et al. Measuring the functional impact of fatigue: initial validation of the fatigue impact scale. Clinical Infectious Diseases 1994;18:S79-83. 
80 Rooney S, McFadyen DA, Wood DL, et al. Minimally important difference of the fatigue severity scale and modified fatigue impact scale in people with multiple sclerosis. Mult Scler Relat Disord 2019;35:158-63.

81 Kinnett-Hopkins D, Adamson B, Rougeau K, et al. People with MS are less physically active than healthy controls but as active as those with other chronic diseases: an updated meta-analysis. Mult Scler Relat Disord 2017;13:38-43.

82 Casey B, Coote S, Galvin R, et al. Objective physical activity levels in people with multiple sclerosis: meta-analysis. Scand J Med Sci Sports 2018;28:1960-9.

83 Schneller MB, Bentsen P, Nielsen G, et al. Measuring Children's Physical Activity: Compliance Using Skin-Taped Accelerometers. Med Sci Sports Exerc 2017;49:1261-9.

84 Baecke JA, Burema J, Frijters JER. A short questionnaire for the measurement of habitual physical activity in epidemiological studies. Am J Clin Nutr 1982;36:936-42.

85 Hertogh EM, Monninkhof EM, Schouten EG, et al. Validity of the modified Baecke questionnaire: comparison with energy expenditure according to the doubly labeled water method. Int J Behav Nutr Phys Act 2008;5:30.

86 Marques JP, Gruetter R. New Developments and Applications of the MP2RAGE Sequence - Focusing the Contrast and High Spatial Resolution R1 Mapping. PLoS One 2013;8:e69294.

87 Thaler C, Faizy T, Sedlacik J, et al. T1- thresholds in black holes increase Clinical-Radiological correlation in multiple sclerosis patients. PLoS One 2015;10:e0144693.

88 McDonald WI, Compston A, Edan G, et al. Recommended diagnostic criteria for multiple sclerosis: guidelines from the International panel on the diagnosis of multiple sclerosis. Ann Neurol 2001:50:121-7.

89 Jensen JH, Helpern JA, Ramani A, et al. Diffusional kurtosis imaging: the quantification of non-Gaussian water diffusion by means of magnetic resonance imaging. Magn Reson Med 2005;53:1432-40.

90 Hansen B, Lund TE, Sangill R, et al. Experimental considerations for fast kurtosis imaging. Magn Reson Med 2016;76:1455-68.

91 Veraart J, Novikov DS, Christiaens D, et al. Denoising of diffusion MRI using random matrix theory. Neuroimage 2016;142:394-406.

92 Kellner E, Dhital B, Kiselev VG, et al. Gibbs-ringing artifact removal based on local subvoxel-shifts. Magn. Reson. Med. 2016;76:1574-81.

93 Andersson JLR, Sotiropoulos SN. An integrated approach to correction for off-resonance effects and subject movement in diffusion MR imaging. Neuroimage 2016;125:1063-78.

94 Hansen B, Jespersen SN. Kurtosis fractional anisotropy, its contrast and estimation by proxy. Sci Rep 2016;6:23999.

95 Eskildsen SF, Ostergaard LR. Quantitative comparison of two cortical surface extraction methods using MRI phantoms. Med Image Comput Comput Assist Interv 2007;10:409-16.

96 Eskildsen SF, Ostergaard LR. Active surface approach for extraction of the human cerebral cortex from MRI. Med Image Comput Comput Assist Interv 2006;9:823-30.

97 Avants BB, Tustison NJ, Stauffer M, et al. The insight toolkit image registration framework. Front Neuroinform 2014;8:44.
98 Abdelhak A, Huss A, Kassubek J, et al. Serum GFAP as a biomarker for disease severity in multiple sclerosis. Sci Rep 2018;8:14798.

99 Gleeson M, Bishop NC, Stensel DJ, et al. The anti-inflammatory effects of exercise: mechanisms and implications for the prevention and treatment of disease. Nat Rev Immunol 2011;11:607-15.

100 Disanto G, Barro C, Benkert P, et al. Serum neurofilament light: a biomarker of neuronal damage in multiple sclerosis. Ann Neurol 2017;81:857-70.

101 El-Sayes J, Harasym D, Turco CV, et al. Exercise-Induced neuroplasticity: a mechanistic model and prospects for promoting plasticity. Neuroscientist 2019;25:65-85.

102 Chan A-W, Tetzlaff JM, Altman DG, et al. Spirit 2013 statement: defining standard protocol items for clinical trials. Ann Intern Med 2013;158:200-7.

103 Schulz KF, Altman DG, Moher D, et al. Statement: updated guidelines for reporting parallel group randomised trials. BMJ 2010;2010:c332.

104 Slade SC, Dionne CE, Underwood M, et al. Consensus on exercise reporting template (CERT): modified Delphi study. Phys Ther 2016;96:1514-24.

105 Brandstadter R, Katz Sand I, Sumowski JF. Beyond rehabilitation: a prevention model of reserve and brain maintenance in multiple sclerosis. Mult Scler 2019;25:1372-8.

106 das Nair R, de Groot V, Freeman J. Beyond current research practice: methodological considerations in MS rehabilitation research (is designing the perfect rehabilitation trial the Holy Grail or a Gordian knot?). Mult Scler 2019;25:1337-47.

107 Dennett R, Madsen LT, Connolly L, et al. Adherence and drop-out in randomized controlled trials of exercise interventions in people with multiple sclerosis: a systematic review and meta-analyses. Mult Scler Relat Disord 2020;43:102169.

108 Bentzen J, Thygesen LC, Flachs EM, et al. Diagnostic delay in multiple sclerosis. Multiple Sclerosis 2010;16:S14-15.

109 Confavreux C, O'Connor P, Comi G, et al. Oral teriflunomide for patients with relapsing multiple sclerosis (tower): a randomised, double-blind, placebo-controlled, phase 3 trial. Lancet Neurol 2014;13:247-56.

110 De Stefano N, Stromillo ML, Giorgio A, et al. Establishing pathological cut-offs of brain atrophy rates in multiple sclerosis. $J$ Neurol Neurosurg Psychiatry 2016;87:93-9.

111 Zivadinov R, Jakimovski D, Gandhi S, et al. Clinical relevance of brain atrophy assessment in multiple sclerosis. Implications for its use in a clinical routine. Expert Rev Neurother 2016;16:777-93.

112 Gyllensten H, Kavaliunas A, Alexanderson K, et al. Costs and quality of life by disability among people with multiple sclerosis: a register-based study in Sweden. Mult Scler J Exp Transl Clin 2018;4:205521731878335.

113 Ness N-H, Schriefer D, Haase R, et al. Differentiating societal costs of disability worsening in multiple sclerosis. J Neurol 2020;267:1035-42.

114 Marck CH, Neate SL, Taylor KL, et al. Prevalence of comorbidities, overweight and obesity in an international sample of people with multiple sclerosis and associations with modifiable lifestyle factors. PLoS One 2016;11:e0148573. 